\title{
A model for the shapes of islands and pits on (111) surfaces of fcc metals
}

\author{
G. T. Barkema and M. E. J. Newman \\ Laboratory of Atomic and Solid State Physics, Cornell University \\ Ithaca, NY 14853-2501.

\begin{abstract}
M. Breeman
Nuclear Solid State Physics, Materials Science Centre, Groningen University

Nijenborgh 4, 9747 AG Groningen, The Netherlands.
\end{abstract}

\begin{abstract}
It is experimentally observed that adsorbate atoms and vacancies on (111) surfaces of fcc metals cluster into islands which are approximately hexagonal, but which on closer inspection turn out to have equilibrium facets which alternate in length $A B A B A B$ around the six sides of the island. By contrast, previous theoretical models for island faceting predict a rotating sequence of three lengths $A B C A B C$ around the island. We propose a new model for the observed shapes, whose physical basis is the variation of the local arrangements of substrate atoms seen by an adsorbate atom. We map our model onto a generalized form of the two-dimensional Ising model having three- as well as two-spin interactions, and estimate using atom-embedding calculations the strengths of these interactions for $\mathrm{Cu}$ adsorbed on a $\mathrm{Cu}(111)$ surface. We then describe a new, highly efficient Monte Carlo technique for calculating the equilibrium crystal shapes of general Ising-type models in two or three dimensions, and apply it to the model in hand. Our results do indeed
\end{abstract}


show alternating facet lengths closely similar to those seen in experiments.

68.35.Bs 05.50.+q

Typeset using REVTEX 


\section{INTRODUCTION}

With the introduction of the technique of scanning tunneling microscopy (STM) it has become possible to study overlayer structures on surfaces in real space with resolution on the scale of single atoms. Of the many interesting surface features this technique has revealed, the ones that will occupy us in the present work are islands of adsorbate atoms on (111) surfaces of metals, and in particular the shapes of these islands, which can be observed either under growth conditions [1], or in thermal equilibrium [Q]1]. During growth, and especially at low temperatures, island shapes can be influenced by the kinetics of the growth process. However, at higher temperatures, kinetic effects are of less importance, and island shapes are governed principally by thermodynamics. There is some experimental evidence that such an equilibrated high temperature regime does exist. In STM experiments on the growth of Pt on Pt (111), Michely and co-workers [3] have compared the shapes of islands formed following sputtering at high temperatures (around 700K) and following high temperature annealing of surfaces sputtered at lower temperatures. Apart from transient effects which are well explained by the details of the experimental technique, the islands are qualitatively very similar, implying that the higher temperatures fall in a regime in which thermal equilibration of a lattice gas of adatoms or vacancies is the dominant effect on island morphology, as opposed to kinetic effects of the sputtering. Another indicative result is that, in this high temperature regime, the islands have a very regular shape, varying little from one island to another, or from large islands to small ones. This behavior is typical of a lattice gas in an equilibrium or near-equilibrium state.

For a thermally equilibrated system, the concept of 'equilibrium crystal shape' (ECS) of adatom islands on surfaces may provide insight into the character of the interactions between adatoms and the interactions between adatoms and the substrate. Since the development of atom-embedding methods like the EAM method of Daw and Baskes [5], the Effective Medium Theory [6], and the Finnis-Sinclair method [7], the calculation of the energetics of larger adatom clusters on surfaces has become feasible. In a recent paper [8], we have 
studied the binding energies and stability of $\mathrm{Cu}$-adatom islands on $\mathrm{Cu}$ (100) and $\mathrm{Cu}(111)$ surfaces by means of the atom-embedding method of Finnis and Sinclair. For islands on $\mathrm{Cu}(111)$ it was found that the binding energies of adatom clusters are dominated by nearestneighbor interactions, with next-nearest-neighbor interactions playing only a minor role. If the binding energy of a cluster were simply proportional to the number of nearest-neighborbonds in the cluster, hexagonal island shapes having all sides of equal length would be preferred. However, the experiments of Michely et al. [3] show hexagonal islands with facets alternating in length around the island. As the experimenters have pointed out, this observation can be understood by noting that there are two possible step-edge (and thus island edge) orientations on a (111) surface of an fcc material; steps either have a $\{100\}$-oriented edge or a $\{111\}$-oriented edge. These two types of edge alternate around the perimeter of a hexagonal surface island, and if the two were equally energetically favorable we would expect the island to be perfectly hexagonal. The fact that the experiments show alternating unequal edge lengths implies that actually one orientation is energetically favored over the other. In our previous study [8], the $\{100\}$-oriented step was found to have a lower energy for $\mathrm{Cu}$ islands on $\mathrm{Cu}$ (111), in agreement with experiment [9]. Experimentally, the reverse seems to be true for $\mathrm{Pt}[3]$, for which the $\{111\}$-oriented step is the energetically favored one, giving rise to surface islands with the opposite orientation to those observed for $\mathrm{Cu}$. In this paper we calculate the ECS of adatom islands on the (111) surface of an fcc metal by means of Monte Carlo simulations of a two-dimensional triangular-lattice Ising-type model. We use a Hamiltonian whose form reflects the physical origin of the different line tensions for the two facet types, and the parameters appearing in the Hamiltonian are fitted to the results of atom-embedding calculations for $\mathrm{Cu}$ islands on $\mathrm{Cu}$ (111) 8]. Although our calculations are all for islands of adatoms, the model is equally well suited to the calculation of the equilibrium shapes of pits in an overlayer of adatoms on a (111) surface. In fact, because of the asymmetric form of the line tension within our model, we expect the ECS for a surface pit to be exactly the same as that for an island of adatoms, for sufficiently large islands, except that it will be rotated by $180^{\circ}$. It is interesting to note that the experiments 
of Michely and co-workers display precisely this phenomenon [3].

In Section II we describe our model. In Section III we give details of the new Monte Carlo technique which we have developed to calculate ECS's for models of this type, and in Section IV we describe the results of our simulations, which show hexagonal surface islands with facets alternating in length $A B A B A B$ around their perimeter, in agreement with experiment. In Section V we give our conclusions.

\section{THE MODEL}

Following a number of previous studies (for example, Ref. [10), we use a form of the Ising model on a triangular lattice in which spins of one sign (say $s=+1$ or 'up' spins) represent sites on the surface which are occupied by adsorbate atoms, and spins of the opposing sign $(s=-1$ or 'down' spins in this case) represent sites which are unoccupied. Surface islands are then represented by domains of up spins in a sea of down spins. Surface pits are represented by domains of down spins in a sea of up. The Hamiltonian includes nearestneighbor ferromagnetic interactions to encourage the aggregation of islands and pits, and we want to add extra terms to make the model duplicate the alternating long and short facet behavior seen in experimental observations. This necessarily means that opposite facets on the same island should have different equilibrium lengths, even though they run in the same direction, which in turn tells us that two-spin interactions will be inadequate to reproduce this behavior in our Ising system; these opposite sides will always have the same line tension and thus the same equilibrium length when only two-spin interactions are present. This is seen clearly in the work of Zia [10] who studied ECS's for Ising models with the most general form of nearest-neighbor two-spin anisotropic interactions on a triangular lattice. The general form of the ECS for these models has three different equilibrium facet lengths rotating in the sequence $A B C A B C$ around the hexagonal island. In all cases, opposite sides have the same length.

In order to understand how the model should be generalized to produce the behavior 
seen in the experimental systems, we consider the physical mechanism which accounts for the alternating equilibrium facet lengths. This mechanism is illustrated in Figure 11. We see that the adsorbate atoms on a triangular lattice can form two different three-atom triangles: the up-pointing ones $\triangle$ and the down-pointing ones $\nabla$. The local environments of the two types of triangles differ because the atoms of one type (in this case, the up-pointing ones) surround an atom in the substrate, whereas those of the other type (down in the figure) do not. This may either increase or decrease the contribution to the binding energy of the up-pointing configuration over that of the down-pointing one, depending on the details of the interaction between adsorbate and substrate atoms. In $\mathrm{Cu}$, for instance, both our calculations and experiment indicated that the up-pointing triangle in the figure should be favored over the down-pointing one, whereas in Pt the situation appears to be reversed.

Within the bulk of an island this difference in energies has no effect, since every adatom belongs to six triangles, three of which are up-pointing and three down-pointing, making every atom energetically equivalent to every other. However, the atoms on the edges of the island belong to only three triangles - either two up and one down, or vice versa, depending on the orientation of the edge. The imbalance in the symmetry of the local environments between these two cases gives rise to a difference in the line tensions of the two types of edges, and thus gives two different equilibrium facet lengths for the island.

To reproduce this behavior in our model we need to introduce a three-spin contribution to the Hamiltonian, which is non-zero only for those sets of three nearest-neighbor sites around a triangle which are all occupied by an adsorbate atom (i.e., for which all three spins are in the up state). Such an interaction term is of the form

$$
\sigma_{i} \sigma_{j} \sigma_{k}+\sigma_{i} \sigma_{j}+\sigma_{j} \sigma_{k}+\sigma_{k} \sigma_{i}+\sigma_{i}+\sigma_{j}+\sigma_{k}+1
$$

where $\sigma_{i}, \sigma_{j}, \sigma_{k}$ are the three Ising spins around the triangle. We multiply this by some interaction energy which takes one of two different values, $K^{\triangle}$ and $K^{\nabla}$, depending on whether the triangle is oriented pointing upwards or downwards, and sum over all trios of spins $\left\langle\sigma_{i} \sigma_{j} \sigma_{k}\right\rangle$ arranged in a triangle. Notice however that in doing this, each pair-wise 
interaction $\sigma_{i} \sigma_{j}$ appears exactly twice, once multiplied by $K^{\triangle}$, and once by $K^{\nabla}$. Thus the two-spin terms in (1) can be accounted for by simply adjusting the nearest-neighbor coupling already appearing in the model according to $J \rightarrow J+K^{\triangle}+K \nabla$. The terms linear in the spins can be rewritten as a simple sum over the lattice $6 \sum_{i} \sigma_{i}$. We will be using conserved order-parameter (COP) dynamics to investigate the formation of islands with diffusing adsorbates, and with a conserved order parameter this linear term is just a constant. Thus, to within a constant, our Hamiltonian takes the form:

$$
H=-J \sum_{\langle i j\rangle} \sigma_{i} \sigma_{j}-K^{\triangle} \sum_{\langle i j k\rangle_{\triangle}} \sigma_{i} \sigma_{j} \sigma_{k}-K^{\nabla} \sum_{\langle i j k\rangle_{\nabla}} \sigma_{i} \sigma_{j} \sigma_{k}
$$

Here the notation $\langle i j k\rangle_{\triangle}$ means that the three sites $i, j, k$ are nearest-neighbors arranged around a triangle oriented upwards, and conversely for $\langle i j k\rangle_{\nabla}$.

Using the atom-embedding method of Finnis and Sinclair [7], we have calculated the binding energies of the ten different 18-atom adsorbate islands shown in Figure 2 for $\mathrm{Cu}$ adsorbed on $\mathrm{Cu}$ (111). We find that our Hamiltonian fits the energies in these calculations to a very good approximation (about $0.012 \mathrm{eV}$ ) with $J=0.144 \mathrm{eV}$ and $K^{\triangle}=-K^{\nabla}=0.0063 \mathrm{eV}$. The effect of the underlying substrate atoms is thus a small one by comparison with the nearest-neighbor coupling, and can to a good approximation be ignored in the calculation of the critical temperature for island formation, which is then given by the corresponding

expression for the ordinary Ising model on a triangular lattice: $\beta J=\arctan \left(1-\mathrm{e}^{\beta J}\right)[11$. When we come to calculate the equilibrium island shape however, the small three-spin term has an important effect on the facet lengths.

\section{THE MONTE CARLO ALGORITHM}

Our strategy for calculating the ECS for our model is to place a certain number of atoms (i.e. up spins) in an island on the triangular lattice and then to allow them to diffuse under some conserved order-parameter Monte Carlo dynamics. We take the resulting distribution of island shapes, superimpose them by identifying their centers of mass, and then average 
over them to find the mean ECS. The exact same method can also be used to calculate the ECS for a pit in the surface.

The simplest COP dynamics is the Kawasaki spin flip, in which adjacent pairs of oppositely-oriented spins are flipped with a probability that depends exponentially on the difference in energies between the states of the model before and after the flip. For the present study however this would be an extremely inefficient dynamics to choose, since the motion of atoms from one part of the island to another is by diffusion in single-latticeparameter steps. Furthermore, the high energetic cost of pulling an atom away from the island edge means that most changes in the shape of the island would take place by edge diffusion, making equilibration still slower. However, since we are not interested in the way in which we reach the ECS, but only in the final result, there is no need to confine ourselves to Monte Carlo moves which are locally order-parameter conserving, like the Kawasaki move, and in fact it turns out that we can do much better by using a non-local move.

The simplest non-local move would be one in which we randomly choose an atom and a vacancy and interchange them, again with an acceptance probability that depends exponentially on the difference in energy between the initial and final states. However, this is also inefficient, since most such moves will involve taking an atom from the bulk of the island and depositing it at some isolated position outside the island. This procedure will have a high energy cost making the acceptance ratio very low. So instead we propose a new algorithm in which, rather than choosing an atom and a vacancy completely at random from the available possibilities, we choose some atoms and vacancies with a higher probability than others, in just such a way, that if we always make the resulting swap of atom and vacancy, detailed balance is exactly obeyed and a Boltzmann equilibrium distribution of states results. In order to describe our algorithm, we need first to define some quantities.

The nearest-neighbor coordination number $N_{i}$ of an adatom on the $i^{\text {th }}$ site is the number of adjacent surface sites also occupied by adatoms:

$$
N_{i}=\sum_{\langle i j\rangle} \delta_{\sigma_{i} \sigma_{j}}
$$


For a vacant surface site, this formula gives the number of adjacent vacancies to that site, and this turns out to be a useful generalization of the coordination number concept, since $N_{i}$ is then the number of satisfied (parallel) bonds to site $i$ in our Ising representation of the problem. We also define the up- and down-triangle coordination numbers $T_{i}^{\triangle}$ and $T_{i}^{\nabla}$ for the $i^{\text {th }}$ site which are the number of up and down triangles respectively of which the site $i$ is a member and on which all the spins are similarly oriented to the one on site $i$ (all occupied by adsorbate atoms, or all vacant). We can write $T_{i}^{\triangle}$ and $T_{i}^{\nabla}$ in the form:

$$
\begin{aligned}
T_{i}^{\triangle} & =\sum_{\langle i j k\rangle_{\triangle}} \delta_{\sigma_{i} \sigma_{j}} \delta_{\sigma_{i} \sigma_{k}}, \\
T_{i}^{\nabla} & =\sum_{\langle i j k\rangle_{\nabla}} \delta_{\sigma_{i} \sigma_{j}} \delta_{\sigma_{i} \sigma_{k}} .
\end{aligned}
$$

Notice that we can equivalently write

$$
\begin{aligned}
T_{i}^{\triangle} & =\frac{1}{4} \sum_{\langle i j k\rangle_{\triangle}}\left(\sigma_{i} \sigma_{j}+\sigma_{j} \sigma_{k}+\sigma_{k} \sigma_{i}+1\right), \\
T_{i}^{\nabla} & =\frac{1}{4} \sum_{\langle i j k\rangle_{\nabla}}\left(\sigma_{i} \sigma_{j}+\sigma_{j} \sigma_{k}+\sigma_{k} \sigma_{i}+1\right) .
\end{aligned}
$$

This becomes useful in the proof of our algorithm.

It now turns out that the correct algorithm for equilibrating the system is to choose an occupied site $i$ with probability $P_{i}$ proportional to $\mathrm{e}^{-2 \beta J N_{i}-4 \beta K^{\triangle} T_{i}^{\triangle}-4 \beta K^{\nabla} T_{i}^{\nabla}}$, and a vacant site $j$ with probability $Q_{j}$ proportional to $\mathrm{e}^{-2 \beta J N_{j}+4 \beta K^{\triangle} T_{j}^{\triangle}+4 \beta K^{\nabla} T_{j}^{\nabla}}$. The correct normalizing factors for the probabilities are simply the sums of these expressions over all occupied and unoccupied sites respectively:

$$
\begin{aligned}
P_{i} & =\frac{\mathrm{e}^{-2 \beta J N_{i}-4 \beta K^{\triangle} T_{i}^{\triangle}-4 \beta K \nabla} T_{i}^{\nabla}}{\sum_{i \mid \sigma_{i}=+1} \mathrm{e}^{-2 \beta J N_{i}-4 \beta K^{\triangle} T_{i}^{\triangle}-4 \beta K \nabla T_{i}^{\nabla}}}, \\
Q_{j} & =\frac{\mathrm{e}^{-2 \beta J N_{j}+4 \beta K^{\triangle} T_{j}^{\triangle}+4 \beta K \nabla} T_{j}^{\nabla}}{\sum_{j \mid \sigma_{j}=-1} \mathrm{e}^{-2 \beta J N_{j}+4 \beta K^{\triangle} T_{j}^{\triangle}+4 \beta K \nabla T_{j}^{\nabla}} .}
\end{aligned}
$$

If we swap two sites chosen in this fashion then detailed balance is almost preserved. The only reason why it isn't is that the probability per unit time of making a particular move now depends on what other moves are possible; a move taking an atom from one part of the 
island's boundary to another, that would be a reasonably probable move in the normal run of things, suddenly becomes very unlikely if there is, for instance, a vacancy in the middle of the island somewhere. The very large binding energy of an atom in this site makes a move that takes a surface atom and fills this vacancy with it overwhelmingly the most likely move in such a case, provided we are a reasonable way below the transition temperature. In order to ensure that the probability of a certain move being made per unit time does not vary in this way, we adjust our time variable $t$ not by unity at each time-step, but by an amount

$$
\frac{1}{\Delta t}=\sum_{i \mid \sigma_{i}=+1} \mathrm{e}^{-2 \beta J N_{i}-4 \beta K^{\triangle} T_{i}^{\triangle}-4 \beta K^{\nabla} T_{i}^{\nabla}} \sum_{j \mid \sigma_{j}=-1} \mathrm{e}^{-2 \beta J N_{j}+4 \beta K^{\triangle} T_{j}^{\triangle}+4 \beta K \nabla T_{j}^{\nabla}} .
$$

This algorithms fulfills the conditions of detailed balance and ergodicity, and therefore samples the Boltzmann distribution corresponding to the Hamiltonian (2). Ergodicity is satisfied, since it is possible ultimately to reach any state, within the COP constraint, by exchanging atoms with vacancies on the lattice. To see that detailed balance is satisfied, we write the rate $T_{i j}$ for a step which exchanges an atom at site $i$ with a vacancy at site $j$ as

$$
T_{i j}=\frac{P_{i} Q_{j}}{\Delta t}=\mathrm{e}^{-2 \beta J N_{i}-4 \beta K^{\triangle} T_{i}^{\triangle}-4 \beta K \nabla T_{i}^{\nabla}} \mathrm{e}^{-2 \beta J N_{j}+4 \beta K^{\triangle} T_{j}^{\triangle}+4 \beta K^{\nabla} T_{j}^{\nabla}}
$$

and the probability of the reverse move as

$$
T_{i j}=\frac{P_{j}^{\prime} Q_{i}^{\prime}}{\Delta t^{\prime}}=\mathrm{e}^{-2 \beta J N_{j}^{\prime}-4 \beta K^{\triangle} T_{j}^{\triangle^{\prime}}-4 \beta K \nabla T_{j}^{\nabla^{\prime}}} \mathrm{e}^{-2 \beta J N_{i}^{\prime}+4 \beta K^{\triangle} T_{i}^{\triangle^{\prime}}+4 \beta K \nabla T_{i}^{\nabla^{\prime}}}
$$

where the primed variables indicate values of the various quantities in the second state. Then the ratio of the rates is

$$
\frac{T_{i j}}{T_{j i}}=\mathrm{e}^{-\beta \Delta E}
$$

where

$$
\begin{aligned}
& \Delta E=2 J\left(N_{i}-N_{i}^{\prime}\right)+2 J\left(N_{j}-N_{j}^{\prime}\right)- \\
& 4 K^{\triangle}\left(T_{i}^{\triangle}+T_{i}^{\triangle^{\prime}}\right)-4 K^{\nabla}\left(T_{i}^{\nabla}+T_{i}^{\nabla^{\prime}}\right)+4 K^{\triangle}\left(T_{j}^{\triangle}+T_{j}^{\triangle^{\prime}}\right)+4 K^{\nabla}\left(T_{j}^{\nabla}+T_{j}^{\nabla^{\prime}}\right) .
\end{aligned}
$$

The terms in $N_{i}-N_{i}^{\prime}$ and $N_{j}-N_{j}^{\prime}$ give the change in energy between the two states, due to the change in the number of satisfied bonds. The terms like $T_{i}^{\triangle}+T_{i}^{\triangle^{\prime}}$ give the change in 
energy due to the triangle interactions. To see this we note that site $i$ is occupied in the first state $\left(\sigma_{i}=+1\right)$ and vacant in the second state $\left(\sigma_{i}^{\prime}=-1\right)$ and then make use of Equation (5) to write

$$
\begin{aligned}
T_{i}^{\triangle}+T_{i}^{\triangle^{\prime}} & =\sigma_{i} T_{i}^{\triangle}-\sigma_{i}^{\prime} T_{i}^{\triangle^{\prime}} \\
& =\frac{1}{4} \sigma_{i} \sum_{\langle i j k\rangle_{\triangle}}\left(\sigma_{i} \sigma_{j}+\sigma_{j} \sigma_{k}+\sigma_{k} \sigma_{i}+1\right)-\frac{1}{4} \sigma_{i}^{\prime} \sum_{\langle i j k\rangle_{\triangle}}\left(\sigma_{i}^{\prime} \sigma_{j}^{\prime}+\sigma_{j}^{\prime} \sigma_{k}^{\prime}+\sigma_{k}^{\prime} \sigma_{i}^{\prime}+1\right) \\
& =\frac{3}{2}+\frac{1}{4} \sum_{\langle i j k\rangle_{\triangle}}\left(\sigma_{i} \sigma_{j} \sigma_{k}-\sigma_{i}^{\prime} \sigma_{j}^{\prime} \sigma_{k}^{\prime}\right) .
\end{aligned}
$$

In a similar way, we can rewrite the $T_{j}^{\triangle}+T_{j}^{\triangle^{\prime}}$ term in (11) as

$$
T_{j}^{\triangle}+T_{j}^{\triangle^{\prime}}=\frac{3}{2}-\frac{1}{4} \sum_{\langle j k l\rangle_{\triangle}}\left(\sigma_{j} \sigma_{k} \sigma_{l}-\sigma_{j}^{\prime} \sigma_{k}^{\prime} \sigma_{l}^{\prime}\right) .
$$

When we take $4 K^{\triangle}$ times the difference of these two expressions, the constant terms cancel, and we are left with precisely the change in the contribution to the energy from the uptriangles. A similar calculation shows the same to be true for the down-triangles, and thus detailed balance is obeyed.

This type of Monte Carlo algorithm is more general than the particular model to which we have applied it in this paper. Similar algorithms can be used to rapidly calculate equilibrium faceting shapes in any conserved order-parameter Ising-type model, and in particular we are not constrained to surface islands; we could equally well use this type of algorithm to calculate three-dimensional crystal shapes. Its use in the calculation of the ECS for simple-cubic and face-centered-cubic crystal shapes with next-nearest-neighbor interactions in three-dimensions has been investigated by Barkema and Holzer [12].

\section{RESULTS}

We have used our Monte Carlo algorithm to calculate equilibrium crystal shapes for islands in our model. The results are shown in Figure 3, for islands consisting of 1875 adatoms on a lattice of 7500 sites. 
Each run takes about ten minutes of CPU time on an IBM RS/6000 workstation. The exact number of Monte Carlo steps used varies from one run to another because the length of the run is measured using the variable time-step of Equation (7). However, on average we find that about $5 \times 10^{6}$ steps are needed for initial equilibration of the island shape, and we run for about a further $10^{7}$ steps to accumulate reasonable statistics on the ECS. The average of the island shape over the run is performed by taking 100 samples of the state of the lattice (one every $\sim 10^{5}$ steps), and calculating the center of mass of the island. From these samples we then calculate the time-averaged occupation of the sites of the lattice over the course of the run (being careful to take account of the variable time-step defined in Equation (7)) with the centers of mass superimposed. The ECS's shown in the figure are the 50 percent occupation contour of the resulting distribution.

The value of $J$ for these runs is fixed at the $0.144 \mathrm{eV}$ found in our atom-embedding calculations for $\mathrm{Cu}$, and we examine the variation of the equilibrium crystal shape with $K$ for $T=300 \mathrm{~K}$ and $T=600 \mathrm{~K}$. The values for $K$ from the atom-embedding calculations fall near the bottom of the range shown in the figure (around $0.01 \mathrm{eV}$ ), where the difference in the equilibrium facet lengths of the islands is quite small. As $K$ is increased, the equilibrium length of the short facets decreases at a greater and greater rate, until, at around $K=0.1$ they appear to vanish completely and the island becomes triangular. It is an open question whether there is a phase transition in the system to the triangular state, and if there is, whether it is a continuous transition.

In the experiments of Michely et al. a roughly constant ratio of the lengths of the long and short sides of islands is observed, with a value of $0.66 \pm 0.05$. Assuming that the nearestneighbor interactions between adatoms in our simulations of $\mathrm{Cu}$ at $600 \mathrm{~K}$ and the experiments on $\mathrm{Pt}$ at $700 \mathrm{~K}$ are comparable, we are able to extract a value of $K^{\triangle}=-K^{\nabla} \approx-0.02 \mathrm{eV}$ for the triangle interaction parameter for Pt. The negative sign here is indicative of the fact that long and short facets in Pt islands are reversed with respect to the facets of the corresponding $\mathrm{Cu}$ islands. The larger numerical value of the interaction corresponds to the greater anisotropy of facet lengths seen in $\mathrm{Pt}$ by comparison with $\mathrm{Cu}$. 


\section{CONCLUSIONS}

We propose both a new model for the anisotropic equilibrium faceting shapes seen in adsorbate islands on (111) surfaces, and also a new non-local Monte Carlo algorithm for calculating ECS's in this or any other conserved order-parameter model for faceting. Using the atom-embedding method of Finnis and Sinclair [7] we have calculated the values of the parameters appearing in the model for $\mathrm{Cu}$ adsorbed on a $\mathrm{Cu}$ (111) surface, and find qualitative agreement with the island shapes seen in the experiments of Michely and coworkers [2- []]. 


\section{REFERENCES}

[1] M. Bott, T. Michely, and G. Comsa, Surf. Sci. 272, 161 (1992).

[2] T. Michely, K. H. Besocke, and G. Comsa, Surf. Sci. Lett. 230, L135 (1990).

[3] T. Michely and G. Comsa, Surf. Sci. 256, 217 (1991).

[4] T. Michely, T. Land, U. Littmark, and G. Comsa, Surf. Sci. 272, 204 (1992).

[5] M. S. Daw and M. I. Baskes, Phys. Rev. Lett. 50, 1285 (1985); M. S. Daw and M. I. Baskes, Phys. Rev. B 29, 6443 (1984).

[6] L. Hansen, P. Stoltzke, K. W. Jacobsen and J. K. Norskov, Phys. Rev. B 44, 6523 (1991).

[7] M. W. Finnis and J. E. Sinclair, Philos. Mag. A 50, 45 (1984).

[8] M. Breeman, G. T. Barkema, and D. O. Boerma, submitted to Surf. Sci.

[9] T. Klas, R. Fink, G. Krausch, R. Platzer, J. Voigt, R. Wesche, and G. Schatz, Europhys. Lett. 7, 151 (1988).

[10] R. K. P. Zia, J. Stat. Phys. 45, 801 (1986).

[11] R. J. Baxter, Exactly Solved Models in Statistical Mechanics, Academic Press, London (1982).

[12] G. T. Barkema and M. Holzer, to be published. 


\section{FIGURES}

FIG. 1. Up-pointing and down-pointing triangles of adatoms on a (111) surface. Notice that the up-pointing triangle has a substrate atom immediately beneath it, whereas the down-pointing one does not. Because of this, interactions between the adatoms and the substrate give rise to a difference between the energies of these two configurations.

FIG. 2. The ten different configurations of eighteen $\mathrm{Cu}$ adatoms on a $\mathrm{Cu}(111)$ surface used to fit the parameters in the Ising Hamiltonian to energies calculated using the atom-embedding method.

FIG. 3. The equilibrium faceting shapes of islands of $\mathrm{Cu}$ adatoms on a $\mathrm{Cu}(111)$ surface calculated within the model described in Section II, using the Monte Carlo method of Section III. The different curves are for different values of the parameter $K$, increasing from outside to inside in the sequence $0.005 \mathrm{eV}, 0.01 \mathrm{eV}, 0.02 \mathrm{eV}, 0.03 \mathrm{eV}, 0.05 \mathrm{eV}$, and for the innermost curve $0.07 \mathrm{eV}$ on the left and $0.1 \mathrm{eV}$ on the right. The curves on the left are the results at $T=300 \mathrm{~K}$ and on the right at $T=600 \mathrm{~K}$. The shapes represent the lines of 50 percent occupation of sites averaged over $\sim 10^{7}$ Monte Carlo steps, as described in Section IV. 


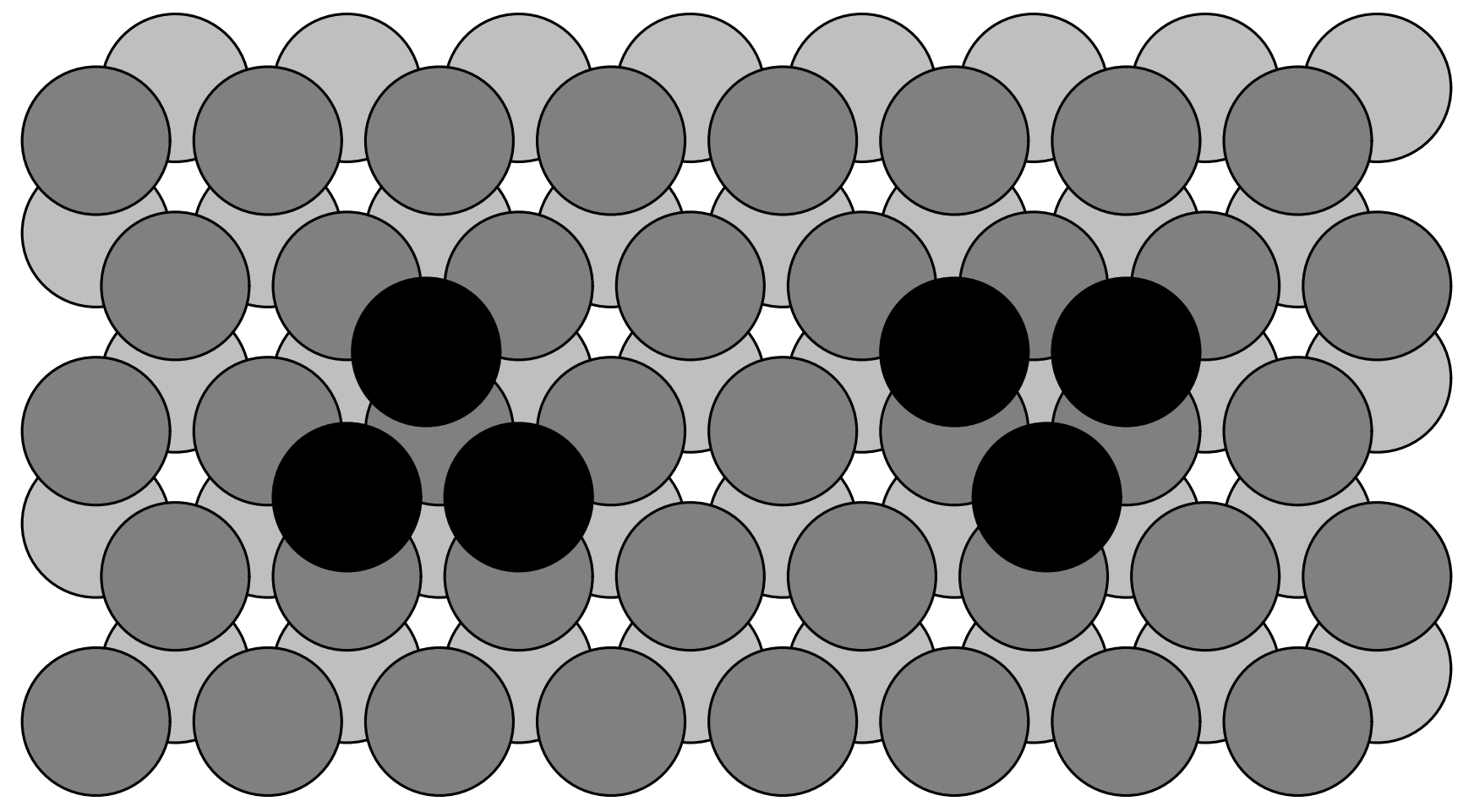

Fig. 1 


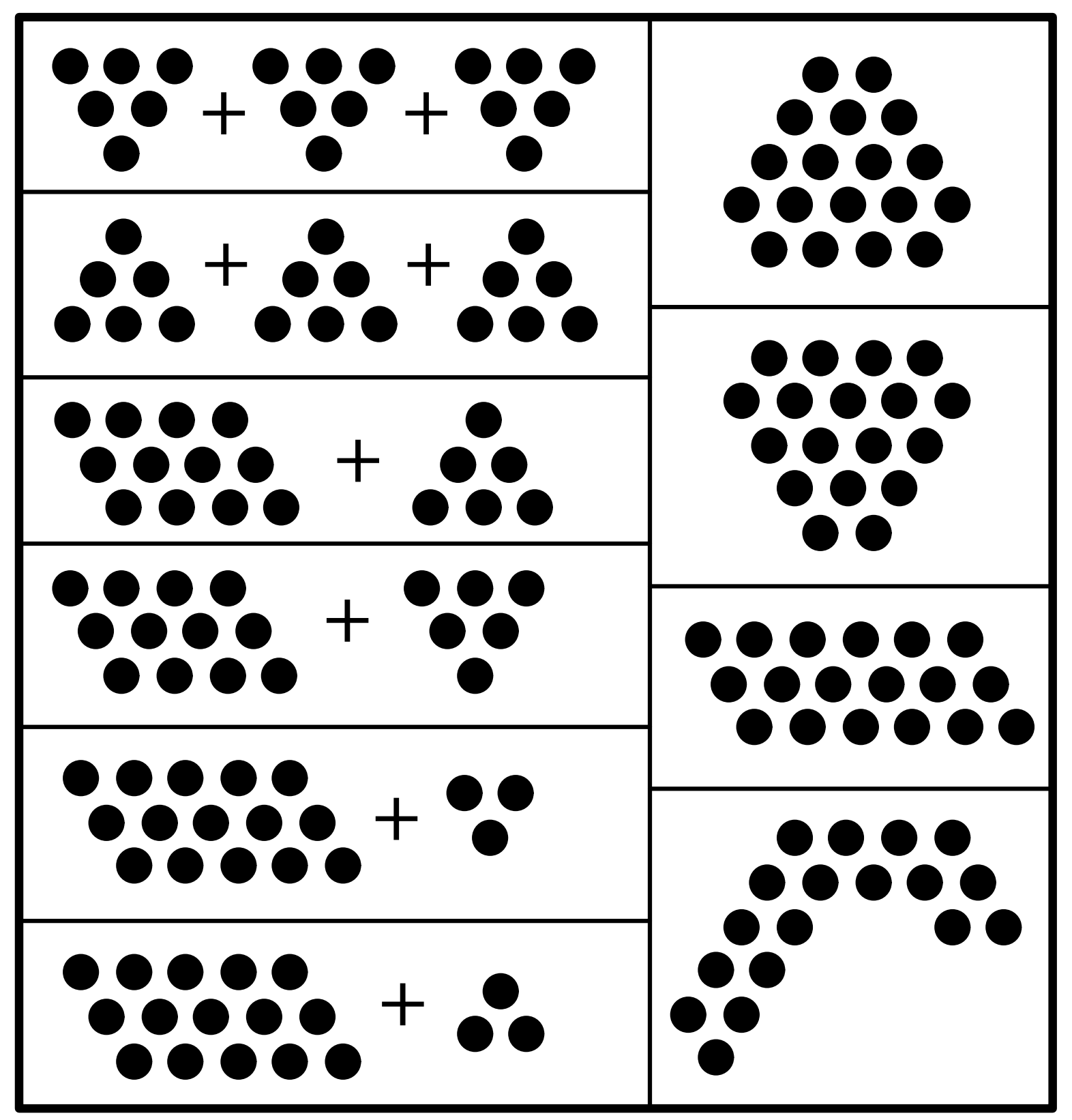

Fig. 2 

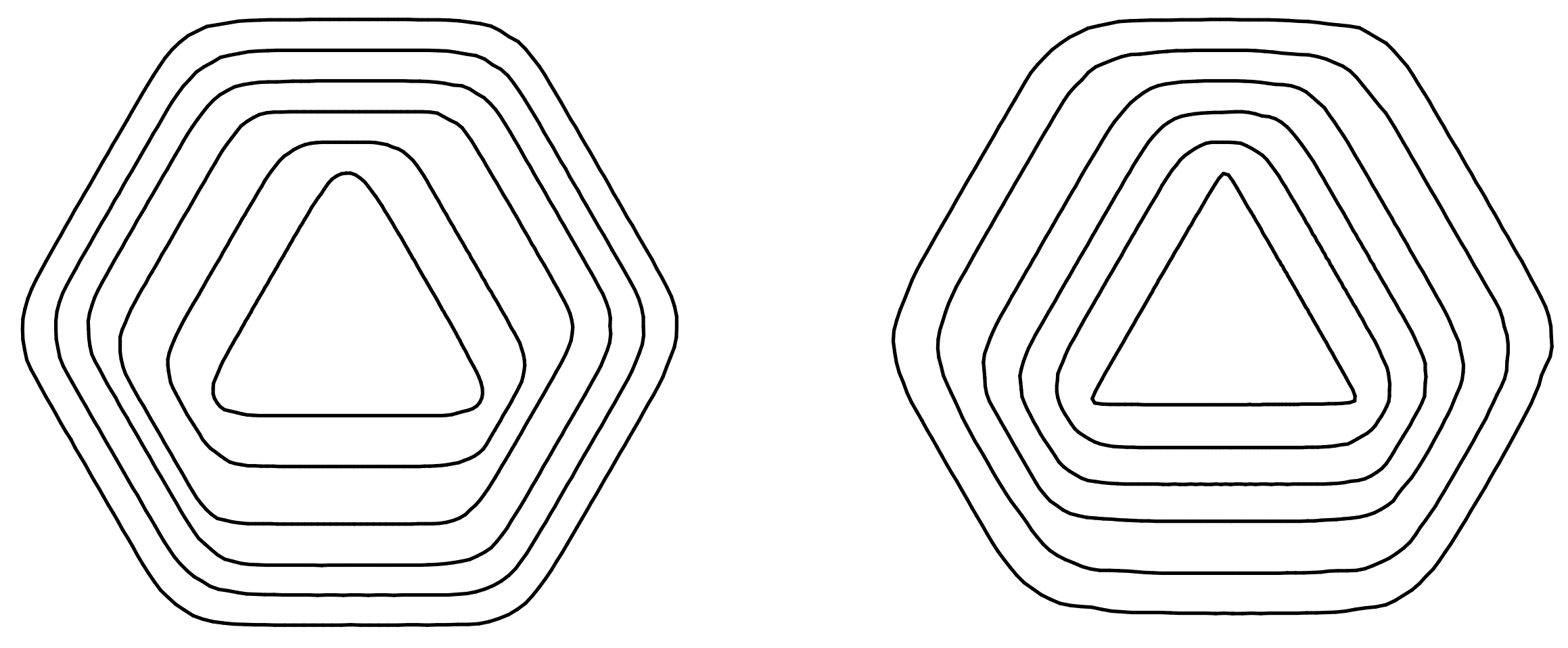

Fig. 3 